\title{
ASSOCIAÇÃO ENTRE ESTADO NUTRICIONAL E PERFIL SOCIOECONÔMICO EM CRIANÇAS DE RECIFE, PERNAMBUCO
}

\section{ASSOCIATION BETWEEN NUTRITIONAL STATUS AND SOCIOECONOMIC PROFILE IN CHILDREN FROM RECIFE, PERNAMBUCO}

\section{ASOCIACIÓN ENTRE ESTADO NUTRICIONAL Y PERFIL SOCIOECONÓMICO EN NIÑOS DE RECIFE, PERNAMBUCO}

Caroline Alencar Dantas ${ }^{1}$, Samara Virginia de Medeiros Albuquerque ${ }^{2}$, Rafaella de Andrade Silva Cavalcanti ${ }^{3}$

Submetido: $18 / 03 / 2020$

Aprovado: 26/06/2020

\begin{abstract}
RESUMO
Introdução: A alimentação de crianças é influenciada pelo perfil socioeconômico da família, sendo um fator de risco para problemas relacionados ao estado nutricional infantil. Objetivo: Avaliar o estado nutricional e o perfil socioeconômico de crianças. Método: Trata-se de um estudo transversal realizado em crianças atendidas numa Organização Não Governamental (ONG) em Recife-PE. Realizou-se a avaliação antropométrica para posterior cálculo do Índice de Massa Corporal (IMC). Foram coletados dados socioeconômicos familiares em entrevista com pais e/ou responsáveis. Resultados: Das 60 crianças avaliadas, o excesso de peso (através do indicador IMC/Idade) prevaleceu nas crianças do sexo masculino. $O$ déficit estatura/idade foi observado em apenas uma criança do sexo feminino. Não houve relação entre o estado nutricional das crianças e os dados socioeconômicos dos pais e/ou responsáveis. Conclusão: $O$ excesso de peso foi mais prevalente entre os meninos e não houve associação do estado nutricional das crianças com os dados socioeconômicos investigados.
\end{abstract}

DESCRITORES: Avaliação Nutricional; Condições Socioeconômicas; Desenvolvimento Infantil.

\section{ABSTRACT}

Introduction: The feeding of children is influenced by the socioeconomic profile of the family, being a risk factor for problems related to children's nutritional status. Objective: To assess the nutritional status and socioeconomic profile of children. Method: This is a cross-sectional study carried out on children assisted at a Non-Governmental Organization (NGO) in Recife-PE. An anthropometric assessment was carried out for later calculation of the Body Mass Index (BMI). Family socioeconomic data were collected in an interview with parents and / or guardians. Results: Of the 60 children evaluated, overweight (using the BMI / Age indicator) prevailed in male children. Height / age deficit was observed in only one female child. There was no relationship between the children's nutritional status and the socioeconomic data of the parents and / or guardians. Conclusion: Overweight was more prevalent among boys and there was no association between children's nutritional status and the socioeconomic data investigated.

DESCRIPTORS: Nutritional Assessment; Socioeconomic conditions; Child development.

Nutricionista graduada pelo Centro Universitário Mauricio de Nassau - Recife-PE

Nutricionista graduada pelo Centro Universitário Maurício de Nassau - Receife-PE

Profa. Dra. do Curso de graduação e pós - graduação em Nutrição da Uninassau e Nutricionista clínica do Hospital Agamenon Magalhães 


\section{RESUMEN}

Introducción: La alimentación de los niños está influenciada por el perfil socioeconómico de la familia, siendo un factor de riesgo para problemas relacionados con el estado nutricional de los niños. Objetivo: evaluar el estado nutricional y el perfil socioeconómico de los niños. Método: Este es un estudio transversal realizado en niños asistidos en una Organización No Gubernamental (ONG) en Recife-PE. Se realizó una evaluación antropométrica para el cálculo posterior del índice de masa corporal (IMC). Los datos socioeconómicos familiares se recopilaron en una entrevista con padres y / o tutores. Resultados: De los 60 niños evaluados, el sobrepeso (utilizando el indicador de IMC / Edad) prevaleció en los niños varones. Se observó déficit de estatura / edad en solo una niña. No hubo relación entre el estado nutricional de los niños y los datos socioeconómicos de los padres y / o tutores. Conclusión: el sobrepeso fue más frecuente entre los niños y no hubo asociación entre el estado nutricional de los niños y los datos socioeconómicos investigados.

DESCRIPTORES: Evaluación nutricional; Condiciones socioeconómicas; Desarrollo infantil. 


\section{INTRODUÇÃO}

A alimentação influencia no estado nutricional e está relacionada com fatores como a cultura, o gênero e a ingestão de alimentos de qualidade na quantidade adequada $^{1,2} \mathrm{e}$, deve estar de acordo com as necessidades de cada fase da vida do indivíduo, principalmente na infância por ser um período de evolução constante e de formação dos hábitos alimentares ${ }^{3}$.

Essa qualidade da refeição oferecida para a criança é influenciada, principalmente, pelo perfil socioeconômico e nível de escolaridade dos pais e/ou responsáveis ${ }^{4}$. Famílias de baixa renda estão mais sujeitas à falta de acesso à determinados alimentos, além de conhecimento escasso sobre alimentação de boa qualidade nutricional ${ }^{4,5}$, o que pode ser considerado um fator de risco para a ocorrência de problemas futuros relacionados ao estado nutricional infantil ${ }^{6}$.

Além disso, a ocupação profissional dos responsáveis também influencia no perfil nutricional das crianças, já que as mulheres, no geral, são as que determinam a alimentação da família, e com o avanço da inserção da mulher no mercado de trabalho, o tempo para a dedicação e cuidados com a alimentação doméstica reduziu, e, consequentemente, a alimentação familiar reduziu a qualidade, o que interfere no estado nutricional dos filhos?

No Brasil, país economicamente emergente, o perfil de consumo alimentar vem sofrendo mudanças ${ }^{8}$, e a transição nutricional reflete diretamente na saúde da população infantil. $O$ resultado principal e mais notável dessa mudança é o excesso de peso ${ }^{9}$, que pode acarretar no surgimento de doenças como Obesidade e Diabetes Mellitus, que são umas das principais causas de morte e hospitalizações no país ${ }^{10}$, além de problemas cardiovasculares e dislipidemias ${ }^{11}$.

As condições socioeconômicas baixas da família (grau de escolaridade, a renda e a ocupação profissional dos pais) são capazes de resultar em um consumo alimentar inadequado das crianças, interferindo diretamente na saúde delas ${ }^{12}$. Estima-se que pessoas de escolaridade e nível socioeconômico menor são mais propensas a desenvolverem Hipertensão Arterial Sistêmica e doenças cardiovasculares ${ }^{13}$. E atualmente há uma prevalência de sobrepeso e obesidade em crianças do Brasil ${ }^{14}$, porém ainda existem diversas crianças que tendem a desenvolver um quadro de desnutrição ${ }^{15}$.

Dessa forma, é essencial avaliar o crescimento e o desenvolvimento de uma criança, assim como o seu estado nutricional, pois mudanças nutricionais interferem nesse crescimento, tanto ponderal quanto estatural. Os parâmetros antropométricos são usados como o método mais importante para essa avaliação na qual são utilizadas as curvas de referência da Organização Mundial da Saúde ${ }^{16}$, que para crianças de cinco a 10 anos, os parâmetros antropométricos ideais são: Peso para idade, Índice de Massa Corporal (IMC) para Idade e Estatura para Idade ${ }^{17}$.

Devido a todos esses fatores é de suma importância realizar a identificação do perfil nutricional de crianças e, a partir disso, programar e efetuar ações de promoção e prevenção de possíveis agravos na saúde, seja desnutrição ou obesidade ${ }^{17}$ já que, consta-se, que a alimentação considerada não saudável é uma causa para o surgimento de Doenças Crônicas Não Transmissíveis (DCNT), a curto e longo prazo ${ }^{18}$. Portanto, o presente estudo teve como objetivo, realizar a associação do perfil socioeconômico ao estado nutricional de crianças.

\section{MÉTODO}

Trata-se de um estudo transversal, realizado em uma Organização Não Governamental (ONG) localizada em Recife-PE, sendo a coleta de dados realizada no período de outubro a novembro de 2019. Foram avaliadas crianças atendidas na ONG, com idades entre cinco a 10 anos, sendo excluídas as crianças portadoras de deficiências mentais ou intelectuais, tais como Autismo, Síndrome de Down e Síndrome de Turner e/ou com problemas físicos que impossibilitaram a avaliação antropométrica.

O projeto de pesquisa foi submetido para análise e aprovado pelo Comitê de Ética em Pesquisa do Centro Integrado de Saúde Amaury de Medeiros (CISAM), pela Plataforma Brasil, de acordo com a Resolução № 466/2012 do Conselho Nacional de Saúde ${ }^{19}$, cujo número do parecer é 3.656.596. Após aprovação do projeto de pesquisa pelo Comitê, os pais ou responsáveis autorizaram, por meio da assinatura do Termo de Consentimento Livre e Esclarecido (TCLE), a criança a participar do estudo.

A avaliação antropométrica foi realizada mediante a aferição do peso, com a utilização de uma balança da marca Welmy ${ }^{\circledR}$, com precisão de $100 \mathrm{~g}$, e da estatura, por meio de uma trena clínica acoplada à parede, da marca Seca ${ }^{\circ}$, com intervalo de medição de 0 a $205 \mathrm{~cm}$ e precisão de $1 \mathrm{~mm}$. Para aferição do peso, as crianças ficaram descalças, apenas com o fardamento utilizado na ONG e foram posicionadas no centro da plataforma da balança. Já para a obtenção da estatura, foi solicita- 
do que as crianças ficassem em pé, descalças, com a postura reta e com os ombros, panturrilhas e calcanhares encostados na parede.

Com os dados obtidos, foi calculado o IMC para diagnosticar o estado nutricional através das curvas de avaliação nutricional de crianças, de ambos os sexos, desenvolvidas pela $\mathrm{OMS}^{16}$, que incluem Estatura por idade, IMC por idade, e Peso por idade. O diagnóstico nutricional foi classificado por meio do Escore-z: Estatura para idade, IMC para Idade, Peso para Idade.

Após o diagnóstico nutricional, foram coletados, através de entrevista com os pais e/ou responsáveis das crianças participantes da pesquisa os dados socioeconômicos: idade dos pais e/ou responsáveis $(<30$ anos; $\geq 30$ anos) pessoas residindo por moradia (duas a quatro pessoas; cinco a oito pessoas); escolaridade dos pais e/ou responsáveis (nove anos ou menos; 12 anos; 16 ou mais) e renda familiar (menos de meio salário mínimo; de meio salário até um salário mínimo; mais de um salário mínimo).

Os riscos para os participantes foram mínimos uma vez que os procedimentos realizados não eram invasivos e foram feitos em local adequado e de forma individualizada para evitar constrangimentos. Os benefícios desse estudo incluíram a divulgação dos resultados da pesquisa para a própria ONG, o que permitiu a identificação daqueles que precisavam de uma maior atenção nutricional, possibilitando intervenção precoce para a promoção da saúde.

O banco de dados e a análise estatística foram realizados através do Statistical Parcktage for the Social Sciences (SPSS) Inc., Chicago, IL, USA, versão 25. As variáveis contínuas foram testadas quanto à normalidade da distribuição, pelo teste de Kolmogorov Smirnof. As variáveis com distribuição normal foram descritas na forma de médias e dos respectivos desvios padrões, foi utilizado o Teste Qui-quadrado para avaliar a associação entre variáveis do estado nutricional das crianças com os fatores socioeconômicos dos seus responsáveis. Na descrição das proporções, a distribuição binomial foi aproximada à distribuição normal, pelo intervalo de confiança de $95 \%$. Sendo considerado estatisticamente significante os valores de "p" inferiores a 5\% para rejeição de hipótese de nulidade.

\section{RESULTADOS}

Foram avaliadas 60 crianças, sendo $58,3 \%(n=38)$ do sexo masculino, com idade prevalente entre cinco a sete anos 70\% ( $n=42)$ (Tabela 1). Na estratificação do estado nutricional das crianças através do indicador IMC/Idade, foi verificado que entre as crianças do sexo masculino, $55,27 \%$ apresentavam excesso de peso, ou seja, classificadas com sobrepeso, obesidade e obesidade grave. Já entre as do sexo feminino, 40,90\% foram classificadas com excesso de peso. (Gráficos 1 e 2).

Com relação ao peso por idade, demonstrado no Gráfico 1, a maioria das crianças do sexo masculino apresentou peso adequado para idade $(n=24 ; 63,15 \%)$, assim como as do sexo feminino ( $n=16 ; 72,73 \%$ ) (Gráfico 2). O déficit de estatura por idade foi observado em apenas um caso entre as meninas ( $n=1 ; 4,55 \%)$ e não houve nenhum caso entre os meninos (Gráficos 1 e 2).

Em relação ao perfil socioeconômico dos pais e/ ou responsáveis, a maioria apresentou idade maior ou igual a 30 anos ( $n=40 ; 66,6 \%)$, com duas a quatro pessoas residindo no domicílio ( $n=33 ; 55 \%)$, com formação até o ensino médio $(n=33 ; 55 \%)$ e renda familiar entre meio e um salário mínimo ( $n=46 ; 76,66 \%)$ (Tabela 1).

Não houve diferença estatisticamente significante entre o estado nutricional das crianças, através do indicador IMC/Idade, quando associado aos dados socioeconômicos dos pais e/ou responsáveis (Tabela 2).

Tabela 1: Perfil socioeconômico das crianças e dos pais e/ou responsáveis participantes do estudo. Recife, Pernambuco, 2019.

\begin{tabular}{|c|c|c|c|}
\hline Variáveis & $\mathrm{n}$ & $\%$ & $\mathrm{IC}_{95 \% *}$ \\
\hline \multicolumn{4}{|l|}{ Sexo das crianças } \\
\hline Masculino & 38 & 63,33 & $48,00-78,65$ \\
\hline Feminino & 22 & 36,67 & $16,54-56,79$ \\
\hline \multicolumn{4}{|l|}{ Idade das crianças } \\
\hline Cinco a sete anos & 42 & 70,00 & $56,14-83,85$ \\
\hline Oito a 10 anos & 18 & 30,00 & $08,83-51,16$ \\
\hline \multicolumn{4}{|l|}{ Idade dos pais e/ou responsáveis } \\
\hline$<30$ & 20 & 33,30 & $12,65-53,95$ \\
\hline$\geq 30$ & 40 & 66,60 & $51,98-81,22$ \\
\hline \multicolumn{4}{|l|}{$N^{\circ}$ de pessoas por moradia } \\
\hline Duas a quatro pessoas & 33 & 55,00 & $38,03-71,97$ \\
\hline Cinco a oito pessoas & 27 & 45,00 & $26,25-63,75$ \\
\hline \multicolumn{4}{|l|}{$\begin{array}{l}\text { Escolaridade dos pais e/ou } \\
\text { responsáveis }\end{array}$} \\
\hline Nove anos ou menos de estudo & 22 & 36,60 & $16,48-56,72$ \\
\hline Doze anos de estudo & 33 & 55,00 & $38,03-71,97$ \\
\hline Dezesseis anos ou mais de estudo & 5 & 08,33 & $00,00-32,55$ \\
\hline \multicolumn{4}{|l|}{ Renda Familiar } \\
\hline Menos de meio $\mathrm{SM}^{\star *}$ & 6 & 10,00 & $00,00-34,01$ \\
\hline De meio salário até um $\mathrm{SM}^{\star *}$ & 46 & 76,66 & $64,43-88,89$ \\
\hline Mais de um SM** & 8 & 13,33 & $00,00-36,88$ \\
\hline
\end{tabular}

Legenda: *Intervalo de confiança de 95\%, **Salário mínimo vigente em 2019 (R\$ 998,00).

Gráfico 1: Classificação do estado nutricional das crianças do sexo masculino através dos indicadores 
IMC/Idade, Peso/Idade e Estatura/Idade. Recife, Pernambuco, 2019.

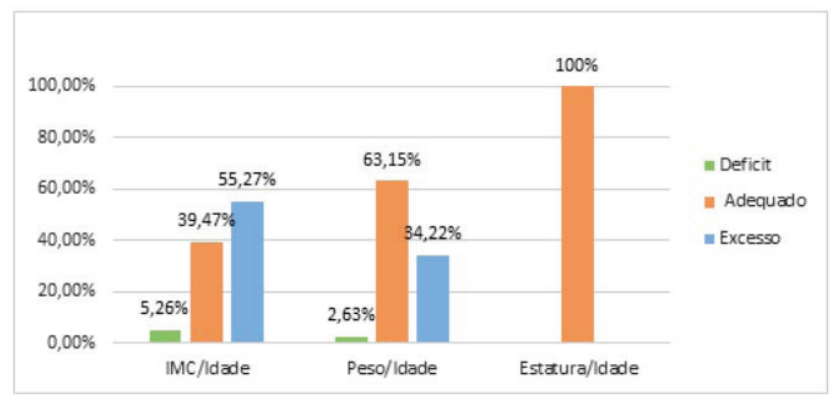

Gráfico 2: Classificação do estado nutricional das crianças do sexo feminino através dos indicadores IMC/ldade, Peso/ldade e Estatura/ Idade. Recife, Pernambuco, 2019.

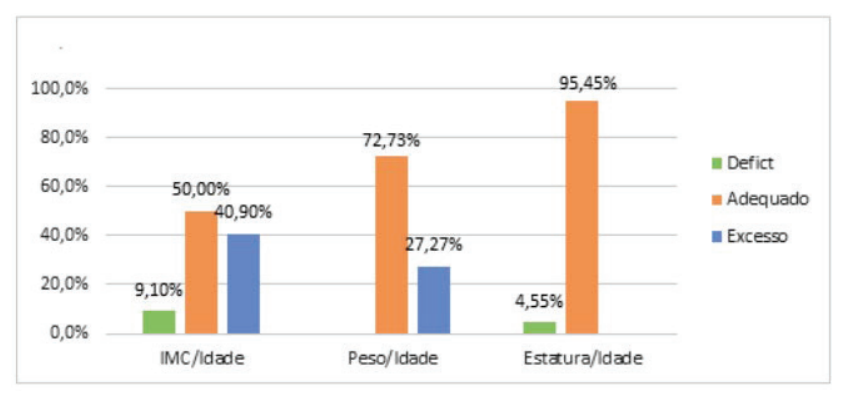

Tabela 2: Associação entre o estrado nutricional das crianças, através do indicador IMC/Idade, com o perfil socioeconômico dos responsáveis. Recife, Pernambuco, 2019.

\begin{tabular}{lllll}
\hline Variáveis & \multicolumn{5}{c}{ Déficit Adequado Excesso $\mathrm{p}^{*}$} \\
\hline Idade dos pais e/ou responsáveis & & & \\
$<30$ & 00 & 06 & 11 & 0,191 \\
$\geq 30$ & 04 & 20 & 19 & \\
$\begin{array}{l}\text { Escolaridade dos pais e/ou } \\
\text { responsáveis }\end{array}$ & 01 & 11 & 09 & \\
Nove anos ou menos de estudo & 03 & 11 & 17 & \\
Doze anos de estudo & 00 & 04 & 00 & 0,331 \\
Dezesseis anos ou mais de estudo & & & & \\
Renda Familiar & & & & \\
Menos de meio SM & & & & \\
De meio até um SM & 00 & 06 & 03 & \\
Mais de um SM** & 04 & 17 & 22 & 0,592 \\
\hline
\end{tabular}

Legenda: *Teste do Qui-quadrado, **Salário mínimo vigente em 2019 (R\$ 998,00)

\section{DISCUSSÃO}

Embora a maioria das crianças do sexo feminino tenham apresentado um estado nutricional de eutrofia segundo o IMC/Idade, 40,9\% apresentou excesso de peso. E também identificado uma elevada prevalência de excesso de peso entre as do sexo masculino, corroborando os achados de Spinelli et al. (2013) ${ }^{20}$ em pesquisa realizada com pré-escolares e escolares de uma escola privada na região central de São Paulo. Esse fato mostra que, mesmo em regiões diferentes, o grau de sobrepeso e obesidade em crianças de cinco a nove anos vem crescendo em todo o país ${ }^{14}$. Uma plausível explicação para isso é que o alto consumo de alimentos calóricos e industrializados nessa faixa etária vem enfrentando um aumento, sendo um fator bastante significante para a obesidade ${ }^{21}$.

Quanto à escolaridade dos pais, não foi identificado associação significativa com o estado nutricional das crianças. Entretanto, estudos realizados em outras populações, como por exemplo, no Paquistão, que mostrou que crianças com pais de nível de escolaridade superior tinham mais probabilidade de ter excesso de peso, em comparação com crianças com pais de nível de escolaridade menor ${ }^{22}$. Assim como em uma pesquisa realizada no México, com escolares de cinco a 10 anos, verificou-se que a maior escolaridade materna é um fator de risco para a obesidade da criança ${ }^{23}$. Outro estudo realizado com alunos de uma escola privada de Brasília apresentou um resultado diferente, demonstrando que o menor grau de escolaridade da mãe da criança está diretamente relacionado com um maior risco para a obesidade ${ }^{24}$. Presume-se que os brasileiros de menor escolaridade compram mais alimentos ultraprocessados por possuírem conhecimento escasso sobre a qualidade desses alimentos ${ }^{4,5}$.

Essas diferenças demonstram que ainda não há uma concordância na literatura no que diz respeito à associação da escolaridade dos pais com o estado nutricional dos seus filhos. Isso porque, acredita-se que um maior nível de escolaridade está relacionado com um maior conhecimento em relação a uma alimentação saudável sendo um fator de proteção ao ganho de peso, contudo, também se pressupõe que leva à uma renda mais alta e com isso, a um acesso com mais facilidade a alimentos de todos os tipos, inclusive altamente calóricos que são fatores de risco para o desenvolvimento da obesidade $^{25}$

Não houve associação estatisticamente significante entre a renda familiar com o estado nutricional das crianças possivelmente por se tratar de uma amostra pequena, ser uma ONG que todas as crianças residem próximas e tem uma similaridade. Porém, em um estudo realizado com alunos no interior de São Paulo, o excesso de peso foi prevalente naquelas, cuja renda familiar 
era próxima a meio salário mínimo, destacando ainda que em média, nove em cada 10 alunos com sobrepeso constituem famílias socialmente vulneráveis ${ }^{26}$. Possivelmente porque as pessoas de menor renda aqui no Brasil, adquirem mais alimentos ultraprocessados por possuírem menos conhecimento dos riscos relacionados ao consumo desses alimentos ${ }^{5}$.

Uma pesquisa realizada com 71 escolares de uma instituição pública de Jaguaribara-CE, também considerou as variáveis socioeconômicas e antropométricas das crianças e foi constatado que as crianças cujo responsáveis possuem renda de dois salários mínimos (cerca de 66,67\%) apresentaram sobrepeso/ obesidade, indicando um ponto interessante pelo fato de $90 \%$ dos estudados terem renda inferior a esse valor indicando que o perfil nutricional pode ser influenciado por diversas faixas de renda ${ }^{27}$.

A quantidade de pessoas que residem no domicílio não influenciou no estado nutricional das crianças. Porém, em estudos realizados em outras regiões do Brasil que abrangeram amostras maiores e diversificadas, foi verificado que há uma influência. Por exemplo, pesquisa realizada no município de Ferros - MG, que utilizou 1322 crianças de áreas diferentes, menores de 10 anos, identificou que $81,3 \%$ dos avaliados que residiam com menos de cinco pessoas, eram estróficos, sendo essa diferença estatisticamente significante quando comparado com os que residiam com mais de cinco pessoas que eram $76,6 \%$.

A grande maioria das crianças apresentou estatura adequada para a idade, no entanto, ainda chama atenção, o fato de ainda existir um caso com baixa estatura. $\mathrm{O}$ que é semelhante ao estudo realizado na área urbana de Guarapuava - PR em 16 escolas púbicas municipais, apesar de ser na região do Sul do país, onde há um padrão de vida e de renda diferentes. Vale ressaltar que nesse mesmo estudo, as crianças com baixa estatura para a idade pertencem às classes econômicas mais baixas $^{29}$. Entende-se que esse fator esteja relacionado com o baixo consumo alimentar da família, levando à perda de peso e diminuição da estatura das crianças ${ }^{30}$. Esse resultado é similar a outros estudos realizados em diversos países como um feito com crianças em favelas urbanas de Bangladesh, onde também foi demonstrado que a menor renda familiar leva a um déficit nutricional, incluindo a baixa estatura ${ }^{31}$.

Segundo o estudo realizado pela Pesquisa de Orçamento Familiar (POF) ${ }^{32}$, as crianças brasileiras acima de cinco anos apresentaram um número maior de excesso de peso e obesidade em diversas regiões do país com rendas variadas, no entanto foi constatado que o déficit de estatura está concentrado em famílias com menor renda familiar, pois o déficit de estatura representa um problema crônico de desnutrição.

É plausível supor que se os resultados obtidos nessa pesquisa possam apresentar limitações, dentre as quais: trata-se de um estudo com uma amostra de 60 crianças, sendo considerado um quantitativo reduzido em comparação a outros estudos; foi realizado em uma ONG onde todas as crianças residem próximas umas das outras, sem diversidades no local onde vivem, assim as crianças da amostra apresentaram similaridade.

\section{CONCLUSÃO}

O excesso de peso através do indicador IMC/ Idade foi mais prevalente entre as crianças do sexo masculino do que entre as do sexo feminino. Não foram identificadas associações significativas entre o perfil socioeconômico dos pais e/ou responsáveis (renda familiar, escolaridade dos responsáveis e número de residentes por domicílio) e o estado nutricional de crianças.

Ressalta-se, ainda, a necessidade de pesquisas adicionais, estudos longitudinais, que englobem um número maior de amostras e que sejam realizadas em áreas diversificadas para constatar relações relevantes entre o perfil socioeconômico familiar com o estado nutricional de crianças. 


\section{REFERÊNCIAS}

1. Pedraza DF, Silva FA, Melo NLS de, Araujo EMN, Sousa CP da C. Estado nutricional e hábitos alimentares de escolares de Campina Grande, Paraíba, Brasil. Cien Saude Colet. 2017;22(2):469-77.

2. Unicef. Como comer bem e melhor - Dicas para promover alimentação saudável e prevenir a obesidade entre crianças e adolescentes. Brasil: UNICEF, 2018.

3. Brognolli JDS, Ceretta LB, Soratto J, Tomasi CD, Ribeiro RSV. Relação entre estado nutricional e conhecimento sobre alimentação adequada e saudável de escolares. Rev Bras Qual Vida. 2018;10(2):1-13.

4. Melo KS, Silva KLGD, Santos MMD. Avaliação do estado nutricional e consumo alimentar de pré-escolares e escolares residentes em Caetés-PE. Revista brasileira de obesidade, nutrição e emagrecimento. 2018;1(2):357-63.

5. Lucia CM Della, Santos LLM, Anunciação PC, Silva BP, Franceschini SCC, Pinheiro-Sant'Ana HM. Perfil socioeconômico e condições de saúde de pré-escolares de duas creches filantrópicas do município de Viçosa, MG. Rev da Assoc Bras Nutr. 2017;8(2):3-11.

6. De Castro Barbosa MI, De Oliveira BR, De Carvalho NA, Martins KA. Educação Alimentar e Nutricional: Influência no comportamento alimentar e no estado nutricional de estudantes. Mundo da Saúde. 2016;40(4):399-409.

7. Melo KM, Cruz ACP, Brito MFSF, Pinho L de. Influence of parents' behavior during the meal and on overweight in childhood. Esc Anna Nery. 2017;21(4):1-6.

8. Moura NC de, Paiva NMN de, Costa J da S, Pereira MR, Carvalho É, Brasil, et al. Biblioteca Virtual em Saúde do Ministério da Saúde. Guia alimentar para a população. 2015;33:1-11.

9. Costa MC, Sousa AF, Lima JTN, Sousa SDF, Ferreira FV, Marques ARA. Estado nutricional, práticas alimentares e conhecimentos em nutrição de escolares. Revista de Atenção à Saúde. 2018;16:12-7.
10. Ministério da Saúde. Diabetes Mellitus. Cadernos de atenção básica. N. ${ }^{\circ}$ 16.Secretaria de Atenção à Saúde. Departamento de Atenção Básica. 2006;187.

11. Oliosa PR, Zaniqueli D, Alvim R de O, Barbosa MCR, Mill JG. Body fat percentage is better than indicators of weight status to identify children and adolescents with unfavorable lipid profile. J Pediatr (Rio J). 2019;95(1):112-8.

12. Oliveira HS de, Bassler TC, Ferro MA e O, Pessalacia JDR, Santos FR dos. Estado nutricional de crianças e adolescentes atendidos em um projeto socioassistencial e educacional. Rev enferm UFPE line. 2017;11:1335-42.

13. Martin R dos S e S, Godoy I de, Franco RJ da S, Martin LC, Martins AS. Influência do nível socioeconômico sobre os fatores de risco cardiovascular. J Bras Med. 2014;102(2):34-7.

14. Ministério da Saúde. Política Nacional de Alimentação e Nutrição. Ministério da Saúde. Secretaria de Atenção à Saúde. Departamento de Atenção Básica. 2013;84.

15. Ew M, An M. Nutrition status and associated factors among children in public primary schools in Dagoretti , Nairobi , Kenya. Afr Health Sci. 2013;13(1).

16. Johnson W, Onuma O, Owolabi M, Sachdev AS. Bulletin of the World Health Organization Stroke: a global response is needed. Bull World Health Organ. 2007;85(9):660-7.

17. Sociedade Brasileira de Pediatria. Avaliação nutricional da criança e do adolescente - Manual de Orientação. São Paulo: Sociedade Brasileira de Pediatria. Departamento de Nutrologia, 2009.

18. Claro RM, Santos MAS, Oliveira TP, Pereira CA, Szwarcwald CL, Malta DC. Consumo de alimentos não saudáveis relacionados a doenças crônicas não transmissíveis no Brasil: Pesquisa Nacional de Saúde, 2013. Epidemiol e Serviços Saúde. 2015;24(2):257-65. 
19. Conselho Nacional de Saúde. Resolução n 466 , de 12 de dezembro de 2012. Dispõe sobre diretrizes e normas regulamentadoras de pesquisas envolvendo seres humanos. Diário Oficial da República Federativa do Brasil, 2013.

20. Spinelli MGN, Morimoto JM, De Freitas APG, De Barros $\mathrm{CM}$, Dias DHS, Pioltine MB, et al. Estado nutricional e consumo alimentar de pré-escolares e escolares de escola privada. Ciência \& Saúde. 2013;6(2):94.

21. Cadamuro SDP, Oliveira DV de, Bennemann RM, Silva ES, Gonçalves JE. Consumo alimentar e avaliação nutricional: caracterização de escolares no município de Maringá - Paraná. Cinergis. 2016;17(2):146-9.

22. Mushtaq MU, Gull S, Shahid U, Shafique MM, Abdullah HM, Shad MA, et al. Family-based factors associated with overweight and obesity among Pakistani primary school children. BMC Pediatr. 2011;11.

23. De Moraes SA, Rosas JB, Mondini L, Martins De Freitas IC. Prevalência de sobrepeso e obesidade e fatores associados em escolares de área urbana de Chilpancingo, Guerrero, México, 2004. Cad Saude Publica. 2006;22(6):1289-301.

24. Giugliano R, Carneiro EC. Fatores associados à obesidade em escolares Factors associated with obesity in school children. J Pediatr. 2004;80(1):17-22.

25. Crispim CA, et al. Conhecimento nutricional de mães de baixo nível socioeconômico participantes e não participantes de um curso de educação nutricional. Rev. Assoc. Bras. Nutr. 2010;3(1).
26. Amistá MJ de M, Silva MV da. Estado nutricional de crianças e adolescentes de um município do interior paulista e vínculo com programas de transferência de renda. Segurança Alimente Nutr. 2015;22(2):721.

27. Araújo CG, Rosa SAM. Perfil socioeconômico e nutricional de escolares em uma instituição pública de ensino em Jaguaribara-CE. Rev APS. 2016;19(4):602-12.

28. Felisbino-Mendes MS, Campos MD, Lana FCF. Avaliação do estado nutricional de crianças menores de 10 anos no município de ferros, Minas Gerais. Rev da Esc Enferm. 2010;44(2):257-65.

29. Bemardi L, Menon MU, Novello D. Prevalência e fatores associados à baixa estatura por idade em escolares. Rev Bras Obesidade, Nutr e emagrecimento. 2018;12(70):165-74.

30. Sawaya AL. Desnutrição: consequências em longo prazo e efeitos da recuperação nutricional. Estud Avancados. 2006;20(58):147-58.

31. Fakir AMS, Khan MWR. Determinants of malnutrition among urban slum children in Bangladesh. Health Econ Rev. Health Economics Review. 2015;5(1):1-11.

32. IBGE - Instituto Brasileiro de Geografia e Estatística. Pesquisa de Orçamentos Familiares 2008-2009: antropometria e estado nutricional de crianças, adolescentes e adultos no Brasil. Ministério da Saúde. 2010;282. 